\title{
(1)
}

\section{ESTUDO DE CASO: ESPIRAIS CONVENCIONAIS X ESPIRAIS DE GRANDE DIÂMETRO*}

\author{
Carlos Magno de Lima Leone ${ }^{1}$ \\ Juliana Cristina Freitas Da Silva² \\ Marcus Vinicius Cunha Lopes ${ }^{3}$ \\ Paulo Henrique Santana 4 \\ Simão Célio de Carvalho 5 \\ Thiago Santos Guedes ${ }^{6}$ \\ William Oliveira Cunha ${ }^{7}$
}

\section{Resumo}

O presente trabalho teve como objetivo comparar o desempenho de processo entre espirais convencionais e espirais de grande diâmetro, na concentração de minérios de ferro. Foram realizadas campanhas de testes para levantar os dados necessários para comparar os dois modelos de espirais em estudo. Os resultados mostraram que as principais variáveis que afetaram a eficiência de separação nas espirais foram a abertura dos lemes e a concentração de sólidos na alimentação. A espiral de grande diâmetro obteve resultados melhores, mesmo sem a utilização de água de lavagem, porém mais testes serão necessários para uma conclusão definitiva.

Palavras-chave: Separação gravimétrica; Espirais concentradoras; Minério de ferro.

\section{CASE STUDY: CONVENTIONAL SPIRALS X LARGE DIAMETER SPIRALS Abstract}

This study aimed to compare the process performance between conventional and large diameter spirals, in the concentration of iron ore. Tests were carried out to obtain the necessary data to compare the two kinds of spirals. The results show that the main variables affecting the separation efficiency in the spirals are the splitters open and the solids concentration in the feed. The large diameter spiral achieved better results, even without the use of wash water, but more tests are needed for a definitive conclusion.

Keywords: Gravimetric separator; Spiral concentrator; Iron ore.

1 Engenheiro de Minas, Doutor em Engenharia de Minas, Gerdau, Miguel Burnier, MG, Brasil.

2 Engenheiro de Minas, Especialista em Gestão de Negócios, Gerdau, Miguel Burnier, MG, Brasil.

Técnico em Mineração, Gerdau, Miguel Burnier, MG, Brasil.

Técnico em Mineração, Gerdau, Miguel Burnier, MG, Brasil.

Engenheiro de Minas, Mestre em Engenharia de Minas, Gerdau, Miguel Burnier, MG, Brasil.

6 Engenheiro Químico, Especialista em Engenharia de Recursos Minerais, Gerdau, Miguel Burnier, MG, Brasil.

7 Técnico em Mineração, Gerdau, Miguel Burnier, MG, Brasil. 


\section{INTRODUÇÃO}

Espirais são equipamentos utilizados para realização de concentração de minérios por método gravítico. Na Unidade de Tratamento de Minérios de Ferro (UTM I) da Gerdau estão instalados bancos de espirais concentradoras do tipo convencional, com duas espiras. Buscando disponibilizar opção tecnológica para viabilizar aumento de produtividade e reduções de custos de investimentos foram realizados estudos comparativos entre o desempenho de espirais convencionais e espirais de grande diâmetro.

\subsection{0bjetivos}

Comparação do desempenho de processo entre espirais convencionais e espirais de grande diâmetro para o minério da mina de Miguel Burnier processado na Unidade de Tratamento de Minérios de Ferro da Gerdau. Avaliação da troca das espirais convencionais por espirais de grande diâmetro.

\subsection{Revisão da Literatura}

A espiral Humphreys teve sua primeira aplicação comercial em 1943, processando areias roladas de cromo [2, appud Hubbard et al. ,1953].

Segundo Arenare et al., [1], a aplicação de espirais concentradoras no processamento de minérios de ferro data da década de 1960, na concentração de hematita especular no Canadá. Desde então, sua aceitação tem sido ampla entre as empresas produtoras de minério de ferro.

A utilização de espirais concentradores é comum em beneficiamento de minério de ferro, principalmente devido aos baixos custos operacionais e de investimentos. Entretanto, o processamento de frações finas $(<0,038 \mathrm{~mm})$ não é possível, tornando outros métodos de concentração mais adequados, como a flotação e a concentração magnética [1].

$\mathrm{Na}$ operação da espiral a polpa de alimentação é introduzida no topo da espiral e à medida que flui para baixo em espiral, as partículas estratificam-se devido ao efeito combinado da força centrífuga, das taxas de sedimentação diferencial das partículas e ao efeito intersticial através do leito. Estes mecanismos são complexos, sendo muito influenciados pela densidade da suspensão e pelo tamanho das partículas [2]. Segundo Lins [3], a água de lavagem é alimentada, sob pressão, na parte central da espiral, através de uma mangueira com furos entre as aberturas que coletam os minerais pesados. Essa água, ao sair sob pressão, centrifuga os minerais leves para a parte periférica da espiral, favorecendo o processo de separação. Essa espiral, com água de lavagem, tem sido usada na etapa de purificação de concentrados.

A capacidade de uma espiral simples é normalmente de $2 \mathrm{t} / \mathrm{h} / \mathrm{espira}$, semelhante à mesa oscilatória, mas ocupando uma área muito menor [3].

O diâmetro das espirais concentradoras tem influência direta na capacidade de alimentação e na faixa granulométrica. Diâmetros maiores permitem uma melhor separação de materiais com faixas de distribuição granulométrica mais amplas. De maneira geral, o beneficiamento de minério de ferro é feito em espirais com diâmetros intermediários [1]. 


\section{MATERIAIS E MÉTODOS}

Foram realizadas campanhas de testes comparativos utilizando-se banco de espirais concentradoras convencionais em operação instalados na UTM 1 e espiral de grande diâmetro adaptada na mesma instalação.

$>$ Características da espiral de grande diâmetro

- Número de voltas: 6

- Número de espiras: 1

- Régua auxiliar para coleta de material concentrado

- Leme coletor de concentrado e médio no final da pista da espira

- Sem água de lavagem

- Diâmetro: $550 \mathrm{~mm}$

> Características da espiral convencional

- Número de voltas: 7

- Número de espiras: 2

- Leme coletor de concentrado em cada volta das espiras

- Com água de lavagem

- Diâmetro: $370 \mathrm{~mm}$

Nos dias 07/01/2014 e 10/01/2014 foram realizados os testes com a espiral de grande diâmetro, alimentada por duas pilhas de ROM em dias diferentes. A primeira pilha com teor de $\mathrm{Fe}$ de $44,4 \%$ e a segunda pilha com teor de Fe de $42,2 \%$.

Entre os dias 04/09/2013 e 12/09/2013 foram realizados os testes com a espiral convencional, alimentada por duas pilhas de ROM em dias diferentes. A primeira pilha com teor de Fe de $37,9 \%$ e a segunda pilha com teor de Fe de $42,0 \%$.

A Figura 1 apresenta o esquema de alimentação e amostragem dos produtos da espiral de grande diâmetro utilizado nos testes.

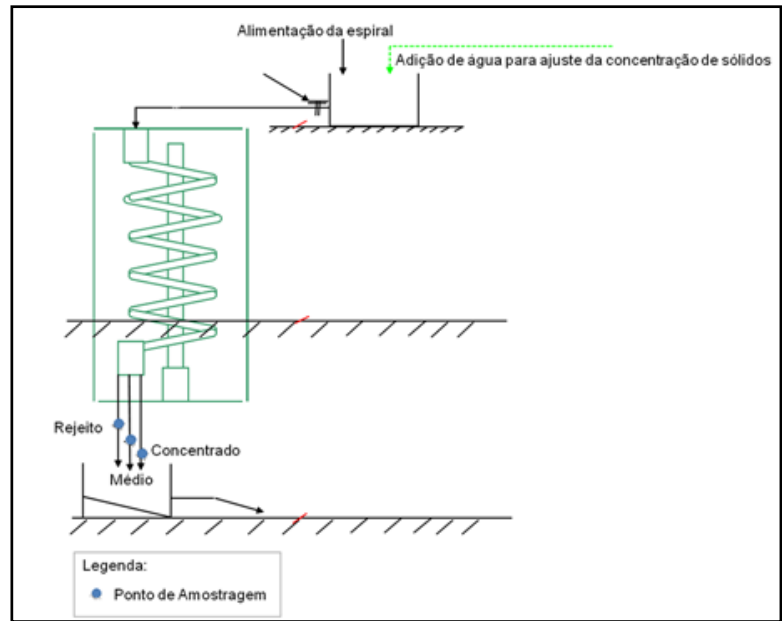

Figura 1. Desenho esquemático da montagem utilizada nos testes com a espiral de grande diâmetro.

Materiais utilizados nos testes e amostragens:

- Espirais completas (grande diâmetro e convencional) incluindo caixas, distribuidor e acessórios;

- Cronômetro;

- Recipientes de coleta: sacos plásticos e baldes;

- Etiquetas para identificação das amostras;

- Balança Marcy;

- Balança digital para até $20 \mathrm{~kg}$. 


\subsection{Procedimentos Gerais}

Para todos os testes (com a espiral de grande diâmetro e convencional) a polpa foi alimentada no distribuidor e medida a concentração de sólidos. Após a medição, a concentração de sólidos foi ajustada para a condição planejada para o teste utilizando as mangueiras auxiliares de água, até a estabilização do circuito. Em seguida foi medida a taxa de alimentação da espiral.

Amostras de alimentação, concentrado e rejeito foram coletadas e enviadas para o laboratório de análises onde foi medida a concentração de sólidos e realizadas as análises químicas.

\subsubsection{Espiral de grande diâmetro}

Os parâmetros definidos para os testes com a espiral de grande diâmetro estão apresentados na Tabela 1.

Tabela 1. Condições dos testes com a espiral de grande diâmetro.

\begin{tabular}{|c|c|}
\hline \multicolumn{2}{|c|}{$\begin{array}{c}\text { Parâmetros Testes Espiral de Grande } \\
\text { Diâmetro }\end{array}$} \\
\hline $\begin{array}{c}\text { Taxa de Alimentação } \\
\text { (t/h/espira) }\end{array}$ & $\begin{array}{c}\text { Concentração de Sólidos } \\
\text { na Alimentação (\%) }\end{array}$ \\
\hline 4 & 40 \\
\hline 4 & 60 \\
\hline 6 & 40 \\
\hline 6 & 60 \\
\hline
\end{tabular}

Para cada um dos parâmetros testados foram adotadas 4 configurações diferentes de posição do leme de concentrado, conforme mostrado na Figura 2. Sendo a configuração 1 com o leme de concentrado na posição mais fechada e variando até a configuração 4, com o leme de concentrado na posição mais aberta.
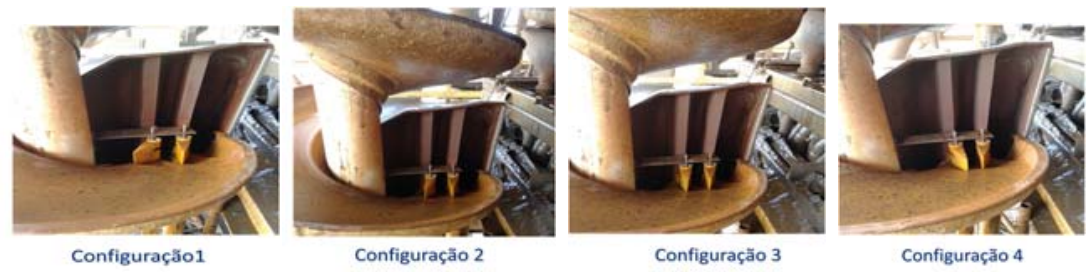

Figura 2. Configuração dos lemes utilizada nos testes com a espiral de grande diâmetro.

\subsubsection{Espiral convencional}

Os parâmetros definidos para os testes com a espiral convencional estão apresentados na Tabela 2.

Tabela 2. Condições dos testes com a espiral convencional.

\begin{tabular}{|c|c|}
\hline \multicolumn{2}{|c|}{ Parâmetros Testes Espiral Convencional } \\
\hline $\begin{array}{c}\text { Taxa de Alimentação } \\
\text { (t/h/espira) }\end{array}$ & $\begin{array}{c}\text { Concentração de Sólidos } \\
\text { na Alimentação (\%) }\end{array}$ \\
\hline 4 & 40 \\
\hline 6 & 60 \\
\hline
\end{tabular}


Foi isolado um banco rougher para realizar os testes com a espiral convencional e utilizada apenas uma das espirais.

Foi adotada apenas uma posição do leme de concentrado, considerando a posição mais aberta, conforme apresentado na Figura 3. Essa é a condição normal de operação da planta.

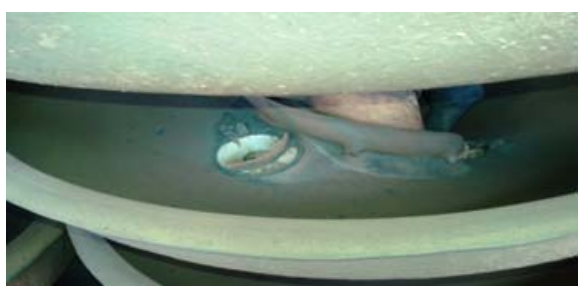

Figura 3. Configuração dos lemes coletores utilizados nos testes com a espiral convencional.

\section{RESULTADOS E DISCUSSÃO}

\subsection{Espiral de Grande Diâmetro}

A Tabela 3 apresenta os resultados dos testes com as espirais de grande diâmetro. A análise dos dados foi realizada utilizando os gráficos apresentados na sequência, obtidos em software de análises estatísticas. As variações apresentadas na tabela entre o real e o objetivo são decorrência de variações na planta. Nesses testes o produto médio foi incorporado ao rejeito.

Tabela 3. Resultados dos testes com espirais de grande diâmetro.

\begin{tabular}{|c|c|c|c|c|c|c|c|c|c|c|c|c|c|}
\hline \multicolumn{14}{|c|}{ RESULTADOS TESTES ESPIRAL DE ALTA CAPACIDADE } \\
\hline \multirow{2}{*}{$\begin{array}{l}\text { Posição } \\
\text { dos Lemes }\end{array}$} & \multicolumn{2}{|c|}{$\begin{array}{c}\text { Taxa de Alimentação } \\
\text { (t/h/espira) }\end{array}$} & \multicolumn{2}{|c|}{\begin{tabular}{|c|} 
Concentração de \\
Sólidos Alimentação
\end{tabular}} & \multirow{2}{*}{$\begin{array}{l}\text { \%Rec. } \\
\text { Mássica }\end{array}$} & \multirow{2}{*}{$\begin{array}{c}\text { \%Rec. } \\
\text { Metalúrgica }\end{array}$} & \multicolumn{3}{|c|}{ \%Fe } & \multicolumn{3}{|c|}{$\% \mathrm{SiO}_{2}$} & \multirow{2}{*}{$\begin{array}{c}\text { IS } \\
\text { Gaudin }\end{array}$} \\
\hline & Objetivo & Real & Objetivo & Real & & & Alimentação & Concentrado & Rejeito & Alimentação & Concentrado & Rejeito & \\
\hline 1 & \multirow{4}{*}{4,0} & 4,7 & \multirow{4}{*}{$40 \%$} & 38,5 & 32,9 & 37,4 & \multirow{4}{*}{56,1} & 63,9 & 33,9 & \multirow{4}{*}{17,2} & 7,5 & 46,9 & 3,4 \\
\hline 2 & & 4,7 & & 47,8 & 54,9 & 61,2 & & 62,5 & 35,0 & & 9,2 & 43,3 & 2,9 \\
\hline 3 & & 4,2 & & 46,6 & 67,3 & 72,9 & & 60,8 & 33,3 & & 11,8 & 45,8 & 2,7 \\
\hline 4 & & 4,3 & & 45,4 & 69,6 & 75,3 & & 60,7 & 31,9 & & 11,6 & 47,0 & 2,8 \\
\hline 1 & \multirow{4}{*}{4,0} & 4,9 & \multirow{4}{*}{$60 \%$} & 50,2 & 36,4 & 44,5 & \multirow{4}{*}{53,3} & 62,4 & 34,5 & \multirow{4}{*}{21,2} & 9,6 & 45,0 & 2,9 \\
\hline 2 & & 4,3 & & 44,6 & 47,7 & 56,7 & & 60,6 & 33,4 & & 12,0 & 46,0 & 2,6 \\
\hline 3 & & 4,5 & & 44,3 & 63,7 & 74,1 & & 59,4 & 31,5 & & 13,6 & 49,8 & 2,6 \\
\hline 4 & & 4,3 & & 43,4 & 70,9 & 79,4 & & 57,1 & 28,3 & & 16,8 & 54,0 & 2,5 \\
\hline 1 & \multirow{4}{*}{6,0} & 6,8 & \multirow{4}{*}{$40 \%$} & 43,9 & 20,6 & 24,6 & \multirow{4}{*}{54,2} & 64,7 & 40,5 & \multirow{4}{*}{20,9} & 6,8 & 39,3 & 3,0 \\
\hline 2 & & 6,4 & & 42,8 & 48,0 & 55,0 & & 63,6 & 35,9 & & 7,7 & 44,8 & 3,2 \\
\hline 3 & & 6,7 & & 43,5 & 59,4 & 66,9 & & 63,5 & 36,9 & & 8,3 & 42,9 & 3,0 \\
\hline 4 & & 7,2 & & 43,9 & 67,8 & 78,5 & & 62,7 & 37,5 & & 8,5 & 41,6 & 2,9 \\
\hline 1 & \multirow{4}{*}{6,0} & 4,7 & \multirow{4}{*}{$60 \%$} & 61,7 & 24,3 & 28,6 & \multirow{4}{*}{53,1} & 62,5 & 39,9 & \multirow{4}{*}{21,7} & 9,1 & 39,0 & 2,6 \\
\hline 2 & & 5,5 & & 64,5 & 28,4 & 32,5 & & 61,3 & 43,9 & & 10,7 & 34,1 & 2,1 \\
\hline 3 & & 6,4 & & 65,3 & 45,1 & 49,0 & & 60,6 & 46,1 & & 11,5 & 30,3 & 1,9 \\
\hline 4 & & 5,7 & & 63,6 & 54,0 & 59,6 & & 60,3 & 44,0 & & 12,2 & 33,5 & 1,9 \\
\hline
\end{tabular}

A Figura 4 apresenta a variação da recuperação metalúrgica média nos testes com as espirais de grande diâmetro em função da posição do leme, da taxa de alimentação e da concentração de sólidos na alimentação. A recuperação metalúrgica aumenta quando o leme de concentrado vai da posição mais fechada (1) para a posição mais aberta (4), devido a maior recuperação mássica obtida quando o leme está na posição mais aberta.

Observa-se pela Figura 4 que, com o aumento da taxa de alimentação das espirais a recuperação diminui. Segundo Arenare et al [1], taxas de alimentação elevadas levam a um aumento da velocidade dos fluxos primário e secundário, levando partículas de finos densos e mistos a serem mantidas em suspensão na zona periférica (de recuperação), além das partículas leves, diminuindo a recuperação metalúrgica. A Figura 5 exemplifica essa teoria.

O aumento da concentração de sólidos na alimentação das espirais reduz a recuperação metalúrgica, conforme observado na Figura 4. 


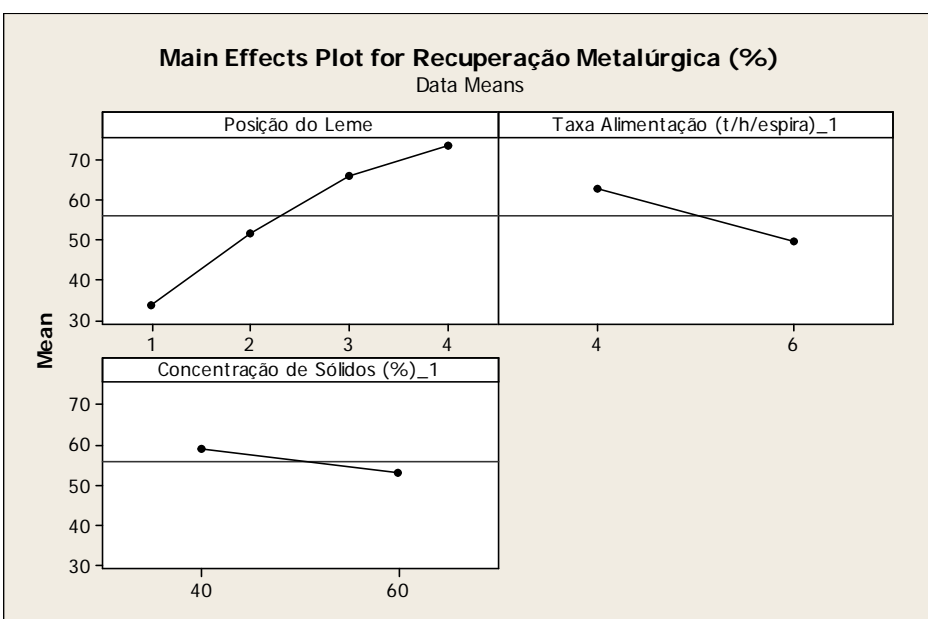

Figura 4. Recuperação metalúrgica nos testes das espirais de grande diâmetro em função da posição do leme, taxa de alimentação e concentração de sólidos.

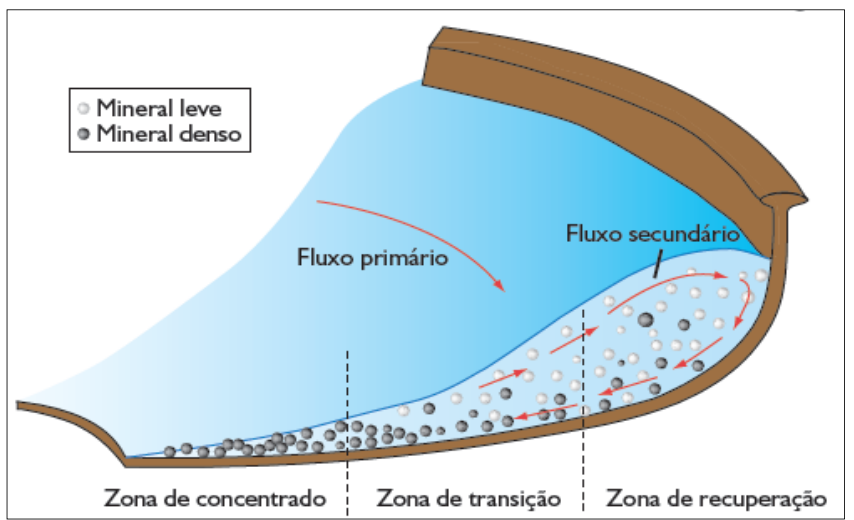

Figura 5. Principais zonas na calha da espiral. (Arenare et al, 2009)

A Figura 6 apresenta a variação do teor de Fe no concentrado (média) nos testes com as espirais de grande diâmetro em função da posição do leme, da taxa de alimentação e da concentração de sólidos na alimentação. O teor de $\mathrm{Fe}$ diminui quando o leme de concentrado vai da posição mais fechada (1) para a posição mais aberta (4). Isso se deve ao fato de que o teor de Fe aumenta gradativamente da borda externa para a interna na espira, influenciado pela força centrífuga atuante e pela densidade mais alta dos minerais de ferro.

Observa-se pela Figura 6 que com o aumento da taxa de alimentação das espirais o teor de $\mathrm{Fe}$ no concentrado aumenta. A explicação é a mesma dada para a recuperação metalúrgica, ou seja, taxas de alimentação elevadas levam a um aumento da velocidade dos fluxos primário e secundário, levando partículas de finos densos e mistos a serem mantidas em suspensão na zona periférica (recuperação), além das partículas leves, aumentando o teor de $\mathrm{Fe}$ no concentrado. A Figura 5 exemplifica essa teoria.

O aumento da concentração de sólidos na alimentação das espirais reduz o teor de Fe no concentrado, conforme observado na Figura 6. A teoria nos diz que o teor de Fe no concentrado deveria ser maior, porém, segundo Arenare et al [1], isso pode ser atribuído à sobrecarga da banda contendo densos, levando estes a serem perdidos no produto leve. 


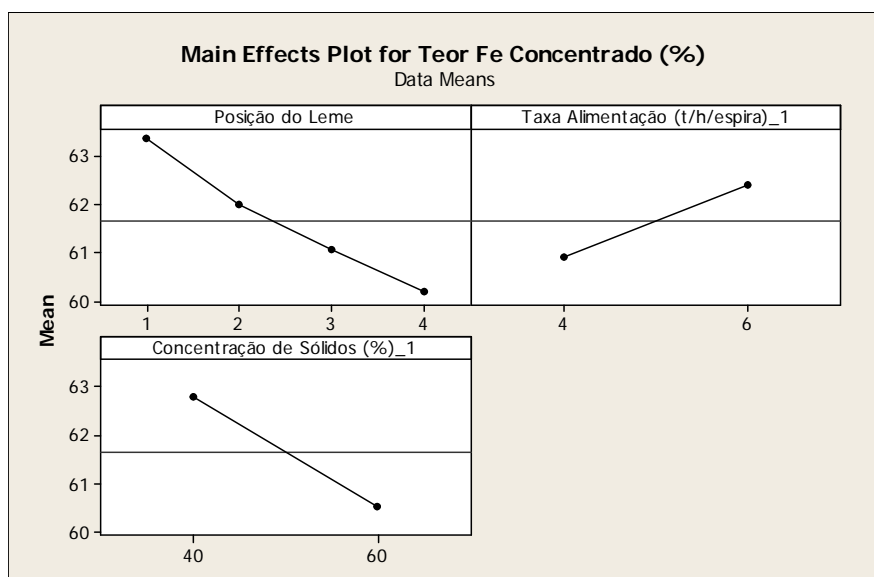

Figura 6. Teor de Fe no concentrado dos testes das espirais de grande diâmetro em função da posição do leme, taxa de alimentação e concentração de sólidos.

A Figura 7 apresenta a variação do Índice de Seletividade (IS) de Gaudin nos testes com as espirais de grande diâmetro em função da posição do leme, da taxa de alimentação e da concentração de sólidos na alimentação. O IS é calculado pela seguinte relação: $\sqrt{ }(\mathrm{R} 1 \times \mathrm{T} 2) /(\mathrm{R} 2 \times \mathrm{T} 1)$ onde: $\mathrm{R}$ é a recuperação para o concentrado da espécie 1 ou 2 e T é a recuperação para o rejeito da espécie 1 ou 2. Nesse caso a espécie 1 é $\mathrm{Fe}$ e $2 \mathrm{SiO}_{2}$, portanto, quanto maior o IS, melhor o resultado da concentração.

O Índice de Gaudin diminui quando o leme de concentrado vai da posição mais fechada (1) para a posição mais aberta (4). Isso se deve ao fato de que a abertura do leme coleta material para o fluxo de concentrado mais próximo da zona de transição, reduzindo a seletividade.

Observa-se pela Figura 7 que com o aumento da taxa de alimentação e concentração de sólidos na alimentação das espirais o IS diminui. Isso se deve ao fato de que o aumento na quantidade de sólidos nas espirais, reduz a eficiência de separação do Fe devido à sobrecarga da banda contendo densos.

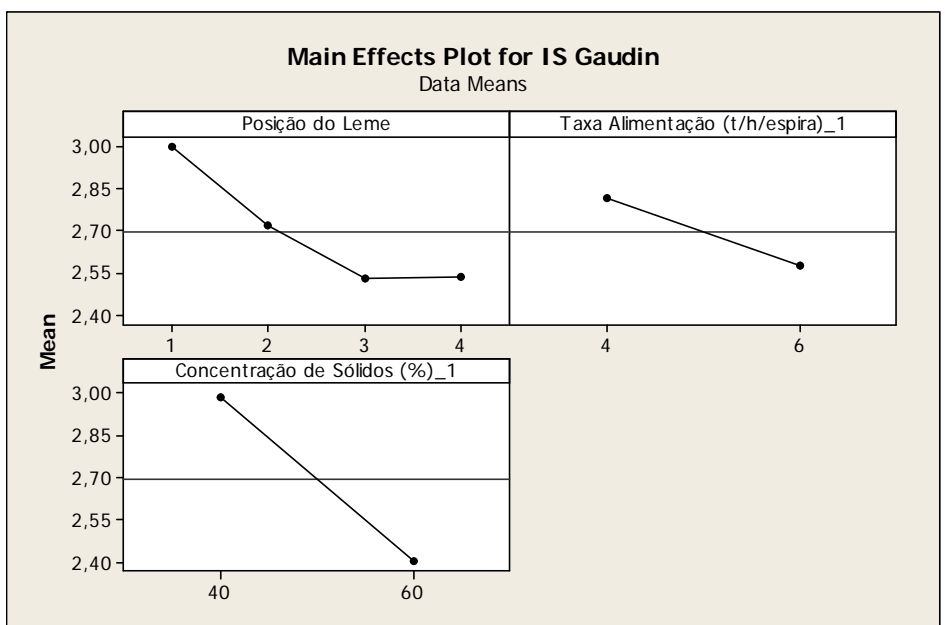

Figura 7. Índice de Seletividade de Gaudin nos testes das espirais de grande diâmetro em função da posição do leme, taxa de alimentação e concentração de sólidos.

As Tabelas 4 a 6 mostram os coeficientes de regressão para as variáveis resposta (recuperação metalúrgica, teor de Fe no concentrado e IS de Gaudin) dos testes nas espirais de grande diâmetro em função das variáveis de entrada (posição do leme, taxa de alimentação e concentração de sólidos na alimentação). 
Para avaliar se as variáveis de entrada são significativas no resultado das variáveis de resposta será analisado o Valor-P nas Tabelas 4 a 6 . Considerando um nível de significância $(\alpha)=0,05$, valores abaixo desse número significam que a variável de entrada (ou a interação das variáveis de entrada) afeta a variável de saída em questão.

Na Tabela 4 observa-se que, apenas a posição do leme e a concentração de sólidos na alimentação das espirais foram variáveis significativas na recuperação metalúrgica.

Tabela 4. Coeficientes de regressão para a Recuperação Metalúrgica nos testes das espirais de grande diâmetro em função da posição do leme, taxa de alimentação e concentração de sólidos.

\begin{tabular}{|c|c|c|c|c|}
\hline Term & Coef & SE Coef & $\mathrm{T}$ & $P$ \\
\hline Constant & 52,4309 & 1,883 & 27,850 & $\odot, \odot \odot \odot$ \\
\hline Posição do Leme & 20,9979 & 2,981 & 7,044 & 0,000 \\
\hline Taxa Alimentação (t/h/espira) & $-5,0458$ & 4,531 & $-1,114$ & 0,294 \\
\hline Concentração de Sólidos (\%) & $-11,1191$ & 2,615 & $-4,253$ & $\odot, \odot \odot 2$ \\
\hline $\begin{array}{l}\text { PRESS }=2611,43 \\
\operatorname{R-Sq}(\text { pred })=48\end{array}$ & $R-S q(a$ & $d j)=87$, & $16 \%$ & \\
\hline
\end{tabular}

Na Tabela 5 observa-se que, apenas a posição do leme foi uma variável significativa no teor de Fe no concentrado. Porém, a concentração de sólidos ficou muito próxima do nível de significância determinado para os testes $(0,05)$ e não deve ser desprezada.

Tabela 5. Coeficientes de regressão para o Teor de Fe no Concentrado nos testes das espirais de grande diâmetro em função da posição do leme, taxa de alimentação e concentração de sólidos.

\begin{tabular}{lrrrr}
\hline Estimated Regression Coefficients for Teor Fe Concentrado (\%) \\
Term & Coef & SE Coef & T & P \\
Constant & 61,7801 & 0,2874 & 214,941 & $\odot, 0 \odot \odot$ \\
Posição do Leme & $-1,0738$ & 0,4551 & $-2,359$ & 0,043 \\
Taxa Alimentação (t/h/espira) & 0,5561 & 0,6918 & 0,804 & $\odot, 442$ \\
Concentração de Sólidos (\%) & $-0,8774$ & 0,3992 & $-2,198$ & 0,056
\end{tabular}

$S=1,00106 \quad$ PRESS $=124,042$

$\mathrm{R}-\mathrm{Sq}=83,92 \% \quad \mathrm{R}-\mathrm{Sq}($ pred $)=\odot, 0 \odot \% \quad \mathrm{R}-\mathrm{Sq}(\operatorname{adj})=73,20 \%$

$\mathrm{Na}$ Tabela 6 observa-se que, apenas a posição do leme e a concentração de sólidos na alimentação das espirais foram variáveis significativas na recuperação metalúrgica.

Tabela 6. Coeficientes de regressão para IS de Gaudin nos testes das espirais de grande diâmetro em função da posição do leme, taxa de alimentação e concentração de sólidos.

\begin{tabular}{|c|c|c|c|c|}
\hline \multicolumn{5}{|c|}{ Estimated Regression Coefficients for IS Gaudin } \\
\hline Term & Coef & SE Coef & $\mathrm{T}$ & $\mathrm{P}$ \\
\hline Constant & 2,58881 & $\odot, \odot 4381$ & 59,087 & $\odot, \odot \odot \odot$ \\
\hline Posição do Leme & $-0,16537$ & $\odot, 06938$ & $-2,384$ & $\odot, 041$ \\
\hline Taxa Alimentação (t/h/espira) & $-\odot, 07410$ & $\odot, 10545$ & $-\odot, 703$ & $\odot, 50 \odot$ \\
\hline Concentração de Sólidos (\%) & $-0,57379$ & $\odot, 06085$ & $-9,430$ & $\odot, 0 \odot \odot$ \\
\hline $\begin{array}{ll}S=0,152594 & \text { PRESS }=0,97220 \\
R-S q=92,49 \% & R-S q(\text { pred })=65\end{array}$ & $8 \% \quad \mathrm{R}-\mathrm{Sq}(\mathrm{a}$ & $d j)=87$, & $49 \%$ & \\
\hline
\end{tabular}


As Tabelas de 4 a 6 mostraram que a posição do leme e a concentração de sólidos na alimentação das espirais foram as variáveis determinantes nos resultados dos testes e que a interação entre essas variáveis não foi significativa para os resultados. A literatura (Arenare et al [2009]) cita que a taxa de alimentação é o fator que mais impacta nos resultados das espirais, o que não foi comprovado nos testes realizados. Mais testes deverão ser executados com as espirais de grande diâmetro, dessa vez com água de lavagem que pode ter impactado nos resultados.

\subsection{Espiral Convencional}

A Tabela 7 ilustra os resultados dos testes com a espiral convencional nas condições de operação industrial. A análise dos dados da tabela será feita utilizando os gráficos apresentados na sequência, comparando com a espiral de grande diâmetro. As variações apresentadas na tabela entre o real e o objetivo são decorrência de variações na planta.

Tabela 7. Resultados dos testes com espiral convencional.

\begin{tabular}{|c|c|c|c|c|c|c|c|c|c|c|c|c|}
\hline \multicolumn{13}{|c|}{ RESULTADOS TESTES ESPIRAL CONVENCIONAL } \\
\hline \multicolumn{2}{|c|}{$\begin{array}{c}\text { Taxa de Alimentação } \\
\text { (t/h/espira) }\end{array}$} & \multicolumn{2}{|c|}{$\begin{array}{c}\text { Concentração de Sólidos } \\
\text { Alimentação }\end{array}$} & \multirow{2}{*}{$\begin{array}{l}\text { \%Rec. } \\
\text { Mássica }\end{array}$} & \multirow{2}{*}{$\begin{array}{c}\text { \%Rec. } \\
\text { Metalúrgica }\end{array}$} & \multicolumn{3}{|c|}{$\% \mathrm{Fe}$} & \multicolumn{3}{|c|}{$\% \mathrm{SiO}_{2}$} & \multirow{2}{*}{ is } \\
\hline Objetivo & Real & Objetivo & Real & & & Alimentação & Concentrado & Rejeito & Alimentação & Concentrado & Rejeito & \\
\hline 4,0 & 4,8 & $40 \%$ & 40,0 & 89,0 & 94,2 & 51,3 & 54,3 & 27,3 & 24,8 & 21,2 & 56,4 & 2,3 \\
\hline 6,0 & 5,4 & $60 \%$ & 62,0 & 65,8 & 76,1 & 50,4 & 58,3 & 35,3 & 26,3 & 15,9 & 45,7 & 2,2 \\
\hline
\end{tabular}

Os dados da espiral de grande diâmetro utilizados na comparação se referem a posição do leme de concentrado mais aberto, uma vez que os testes com a espiral convencional da planta utilizaram o leme nessa posição.

A Figura 10 mostra os resultados calculados do Índice de Seletividade de Gaudin para a espiral convencional e de grande diâmetro em função da taxa de alimentação (a) e da concentração de sólidos (b). A Figura 10 mostra que a eficiência de separação na espiral de grande diâmetro foi mais sensível à concentração de sólidos, mas, tanto para a taxa de alimentação quanto para a concentração de sólidos, a espiral de grande diâmetro obteve, aparentemente, resultados melhores. Porém, como a configuração das espirais de grande diâmetro e convencional são diferentes (água de lavagem, posição dos lemes e coleta de produtos) é necessário realizar mais testes para uma conclusão definitiva.

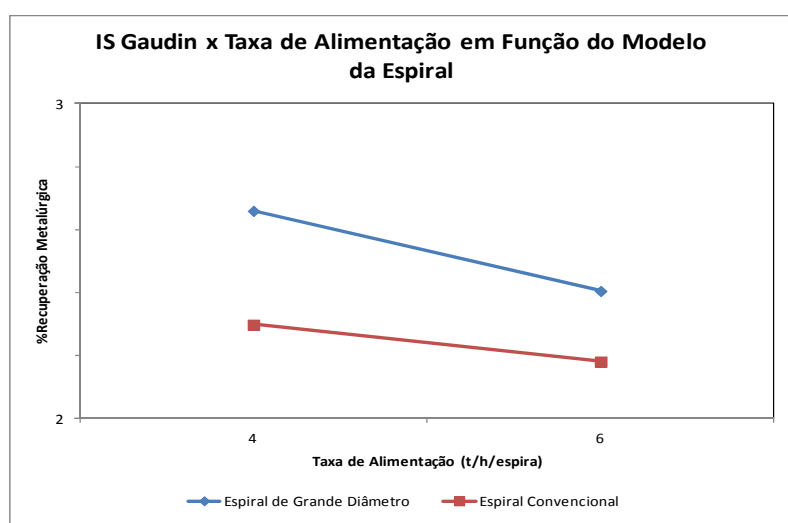

(a)

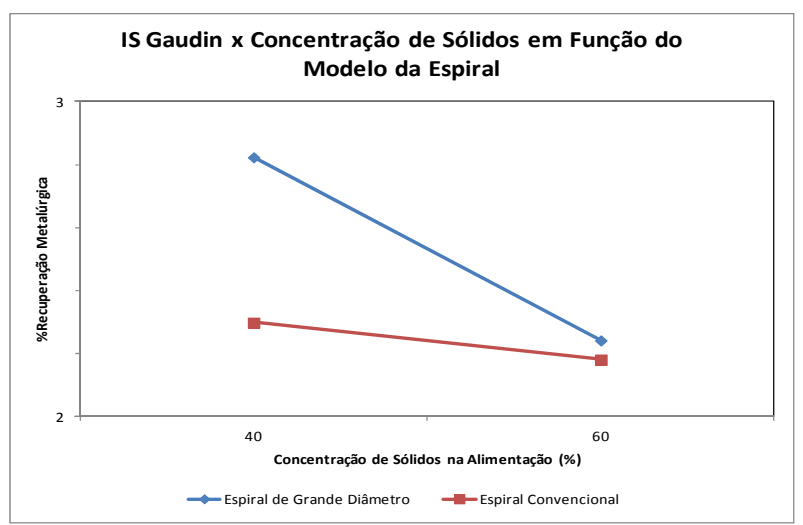

(b)

Figura 10. Índice de seletividade de Gaudin da espiral x taxa de alimentação (a) e concentração de sólidos (b) em função do modelo da espiral. 


\section{CONCLUSÃO}

As principais variáveis que afetaram a eficiência nas espirais de grande diâmetro foram a posição do leme e a concentração de sólidos na alimentação. Os dados obtidos nos testes com espiral de grande diâmetro foram melhores do que aqueles com as espirais convencionais, porém a configuração das espirais é diferente. Testes com a espiral de grande diâmetro e com água de lavagem serão realizados para avaliar a influência desse parâmetro na concentração gravimétrica por espirais, além da utilização de diferentes condições nas espirais convencionais.

\section{REFERÊNCIAS}

1 Arenare, D.S., Rodrigues, O.M.S., Araujo, A.C., Viana, P.R.M., Espirais concentradoras no tratamento de minérios de ferro: uma breve revisão, Tecnol. Metal. Mater., abr.-jun, 2009, v. 5, n. 4, São Paulo, p. 224-228.

2 Wills, B.A., Napier-Munn, T.J. Gravity concentration In: Wills' Mineral processing technology - An Introduction to the Practical Aspects of Ore Treatment and Mineral, 7. ed., Elsevier Science \& Technology Books, October 2006. p. 236-238.

3 Lins, F.A.F. Comunicação Técnica elaborada para a $4^{a}$ Edição do Livro de Tratramento de Minérios, Dezembro 2004, p. 241 a 268. 